\section{Neutering and early- onset urinary incontinence in bitches under primary veterinary care in the UK: A VetCompass cohort study}

\section{Camilla Pegram ${ }^{1}$, Dave Brodbelt ${ }^{1}$, David Church ${ }^{1}$, Jon Hall ${ }^{2}$, Laura Owen ${ }^{3}$, Yu-Mei Chang ${ }^{1}$, Dan O'Neill ${ }^{1}$}

\footnotetext{
1 The Royal Veterinary College, Hatfield, United Kingdom

2 University of Edinburgh, Edinburgh, United Kingdom

3 University of Cambridge, Cambridge, United Kingdom
}

\section{OBJECTIVES}

Urinary incontinence commonly affects bitches and has been attributed to neutering. This study aimed to evaluate associations between neutering and early-onset ( $\leq 8$ years) urinary incontinence (EUI) in bitches under primary veterinary care in the UK.

\section{METHODS}

This retrospective cohort study followed bitches within VetCompass born from January 1st 2010 to December 31st 2012 over time until 31st March 2018. EUI cases were identified and the incidence risk over the study period was calculated. Cox regression modelling evaluated the hazard of neutering for EUI outcome a) from the date of birth for all bitches both neutered and entire and b) from the date of neutering for just the neutered subset of bitches. Confounding variables considered included breed, bodyweight and veterinary practice group.

\section{RESULTS}

The study included 493 EUI cases and 72478 non-cases. Incidence risk was $0.68 \%$ (95\% confidence intervals 0.62 to 0.74$)$. After accounting for confounding factors, increased hazard of EUI was identified in a) neutered bitches, with the effect increasing with age and b) bitches neutered prior to 6 months within the first two years following neuter. In both models, increased hazard was additionally associated with increasing bodyweight and the Irish setter had the highest hazard of EUI by breed.

\section{STATEMENT (CONCLUSIONS)}

Neutering and early-age neuter ( $<6$ months) were identified as major time-dependent factors associated with increased EUI. Although neutering decision-making cannot be just based on EUI risk alone, the reduced time to EUI diagnosis in early-neutered bitches is a consideration, particularly in high-risk breeds and bitches of larger bodyweights.

\section{Effect of treatment of hyperthyroidism on serum SDMA concentrations and the utility of SDMA as a marker of chronic kidney disease in hyperthyroidism}

\section{Emma Tarrant, Tim Williams}

Department of Veterinary Medicine, University of Cambridge, Cambridge, United Kingdom

\section{OBJECTIVES}

To compare serum SDMA concentrations in hyperthyroid cats before and after medical treatment and evaluate the sensitivity and specificity of serum SDMA and creatinine concentrations for concurrent chronic kidney disease (CKD) in hyperthyroidism.

\section{METHODS}

Hyperthyroid cats that commenced medical treatment and in which euthyroidism was achieved $(n=12)$ were identified from samples submitted to Central Diagnostic Services, Cambridge. Cats that were azotaemic before ( $n=3$ ) and/or after treatment ( $n=4$, up to 8 months) were classified as having concurrent CKD. SDMA concentrations (measured in stored frozen samples) in hyperthyroid cats pre- and post-treatment were compared using Wilcoxon signed-rank test. Sensitivity and specificity of elevated pre-treatment serum creatinine (>153 $\mu \mathrm{mol} / \mathrm{L})$ and SDMA concentrations (>14 $\mu \mathrm{g} / \mathrm{dL})$ for concurrent CKD were calculated. Data are presented as median [range].

\section{RESULTS}

Serum SDMA concentrations did not change significantly after 1 month (pre-treatment 14 [7-21] $\mu \mathrm{g} / \mathrm{dL}$ vs. 1 month post-treatment 17 [11-29] $\mu \mathrm{g} / \mathrm{dL}, \mathrm{n}=6$; $\mathrm{P}=0.248$ ] or 3-8 months of treatment (3-8 months post treatment 17 [9-30] $\mu \mathrm{g} / \mathrm{dL}, \mathrm{n}=10 ; \mathrm{P}=0.167$ ). Sensitivity and specificity of elevated pre-treatment serum SDMA 